\title{
COVID-19 with rheumatic diseases: a report of 5 cases
}

\author{
Chuanfang Cheng ${ }^{1} \cdot$ Chuanjing $\mathrm{Li}^{1} \cdot$ Tao Zhao ${ }^{1} \cdot$ Jing Yue ${ }^{1} \cdot$ Fang Yang ${ }^{2} \cdot{\text { Yimin } \text { Yan }^{1,2,3} \text { (D) Xiaoan Liu }}^{4,5}$
}

Received: 23 April 2020 /Revised: 23 April 2020 / Accepted: 5 May 2020/Published online: 14 May 2020

(C) International League of Associations for Rheumatology (ILAR) 2020

\begin{abstract}
The coronavirus disease 2019 (COVID-19), the result of an infection with the new virus, SARS-CoV-2, is rapidly spreading worldwide. It is largely unknown whether the occurrence of COVID-19 in patients with rheumatic immune diseases has some specific manifestations, or makes them more prone to rapidly progress into severe COVID-19. In this case report, we describe the clinical features of 5 rheumatic immune disease patients with the concomitant presence of COVID-19. Amongst these patients, 4 had rheumatoid arthritis (RA) and 1 had systemic sclerosis (SSc). Two patients had a history of close contact with a COVID-19 patient. The age of the patients ranged between 51 and 79 years. Fever (80\%), cough (80\%), dyspnea (40\%), and fatigue (20\%) were the most common presenting symptoms. Laboratory investigations revealed leukopenia and lymphopenia in 2 patients. In all the patients, chest computerized tomography (CT) revealed patchy ground glass opacities in the lungs. During the hospital stay, the condition of two patients remained the same (i.e., mild COVID-19), two patients progressed to the severe COVID-19, and one patient worsened to the critically ill COVID19. These patients were treated with antiviral agents for COVID-19, antibiotics for secondary bacterial infections, and immunomodulatory agents for rheumatic immune diseases. All the patients responded well, were cured of COVID-19, and subsequently discharged.
\end{abstract}

Keywords Coronavirus · COVID-19 · Rheumatic immune disease $\cdot$ SARS-CoV-2

\section{Introduction}

A local outbreak of a novel coronavirus $(\mathrm{CoV})$ disease, i.e., COVID-19 has briskly turned into a global healthcare

Chuanfang Cheng and Chuanjing Li contributed equally to this work.

Yimin Yan

yanyimin180@163.com

$\triangle$ Xiaoan Liu

liuxaxg@163.com

1 Department of Rheumatology and Immunology, Xiaogan Hospital Affiliated to Wuhan University of Science and Technology, The Central Hospital of Xiaogan, Xiaogan 432000, Hubei, China

2 Medical College of Wuhan University of Science and Technology, Wuhan 430071, China

3 Rheumatology and Immunology, Xiaogan Hospital Affiliated to Wuhan University of Science and Technology, No. 6 Plaza Street, Xiaogan 432000, Hubei, China

4 Department of Rehabilitation Medicine, Xiaogan Hospital Affiliated to Wuhan University of Science and Technology, The Central Hospital of Xiaogan, Xiaogan 432100, Hubei, China

5 Department of Rehabilitation Medicine, Xiaogan Hospital Affiliated to Wuhan University of Science and Technology, No. 6 Plaza Street, Xiaogan 432000, Hubei, China emergency [1]. An abrupt augmentation of SARS-CoV-2 infection may be related to an "inflammatory storm," in which the cytokines participate, and result in multiple organ failure and death $[2,3]$. The patients with rheumatic immune diseases have immune dysfunction and are often on glucocorticoid and immunosuppressant therapy, which in turn predispose them to an increased risk of contracting SARS-CoV-2 infection. Few studies have described the concomitant presence of COVID19 in patients with rheumatic diseases. To add to the growing literature, we report the clinical characteristics, radiological findings, treatment, and prognosis of 5 patients of rheumatic disease presenting with COVID-19.

\section{Case reports}

Amongst the 568 patients admitted in The Central Hospital of Xiaogan, between January 16 and March 28, 2020, due to novel $\mathrm{CoV}$ pneumonia, 5 patients had a past history of rheumatic diseases, thus, representing $0.88 \%$ of all the COVID-19 patients admitted in our hospital. The diagnosis of COVID-19 was as per the criteria recommended by the Chinese Center for Disease Control (CDC), which included a positive real-time reverse transcription PCR testing for SARS-CoV-2 (rRT- 


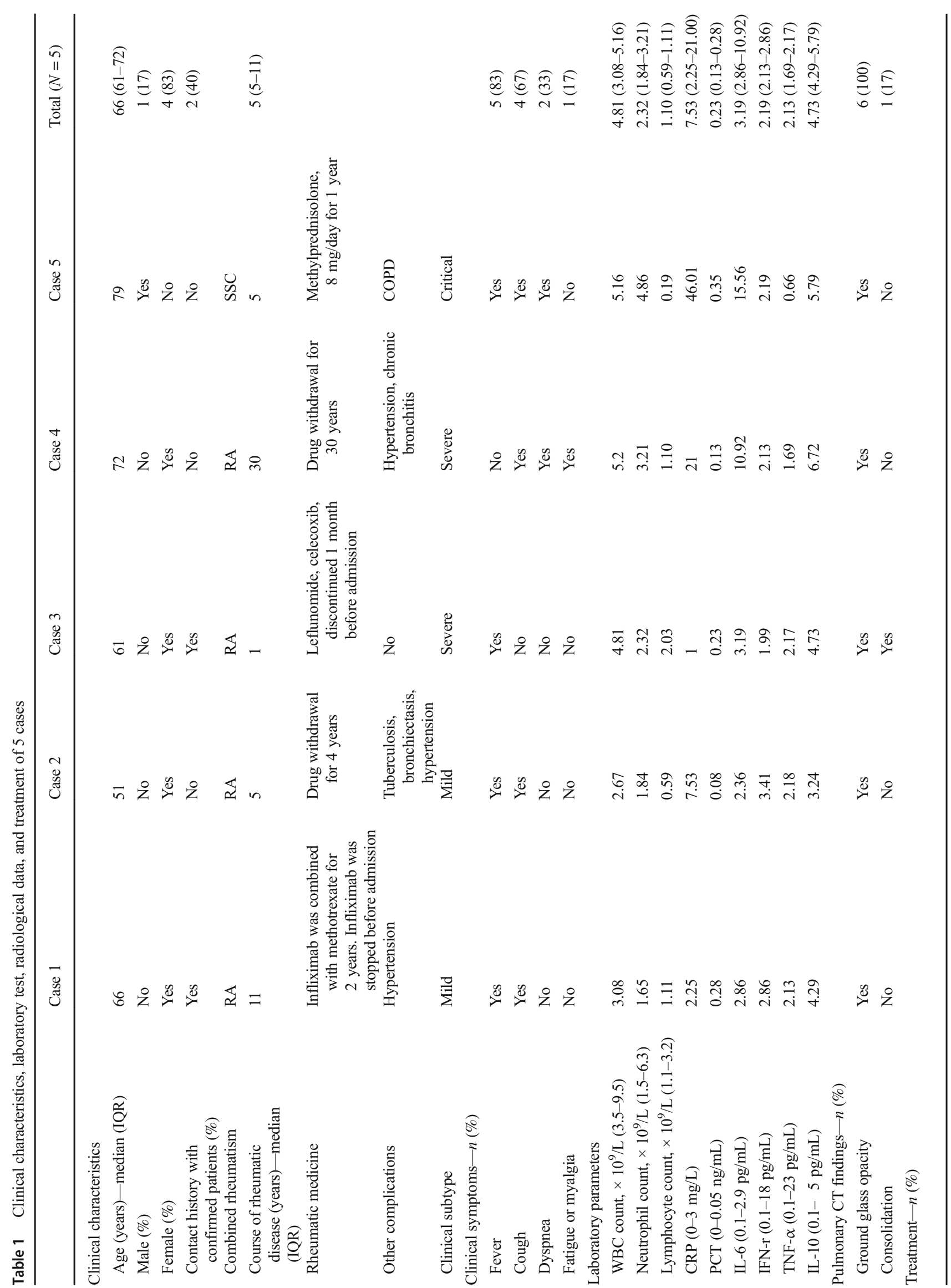




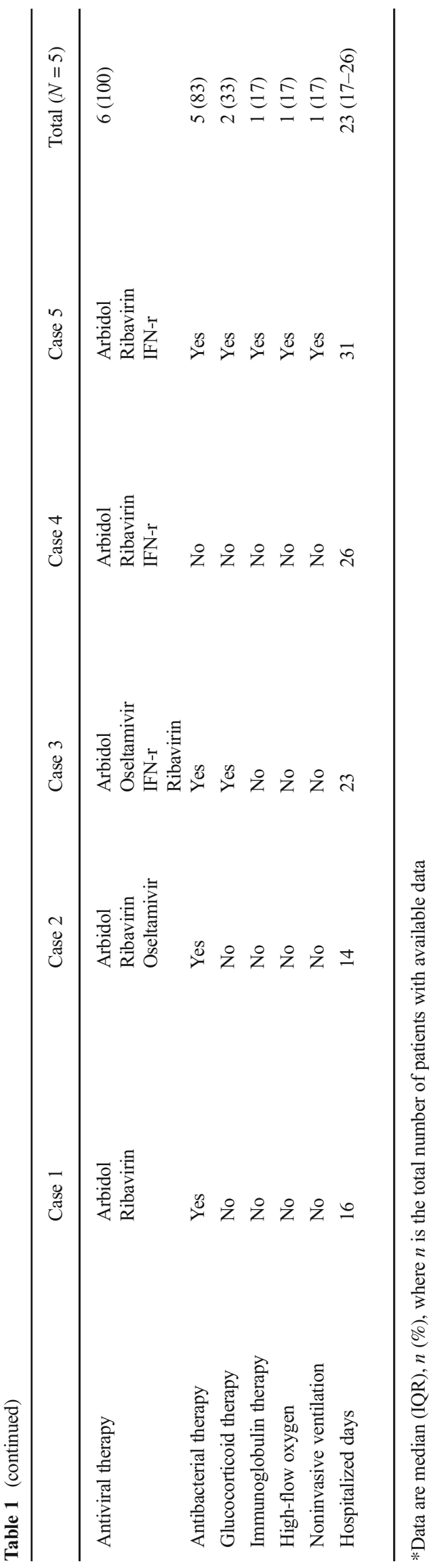

PCR) [4]. The characteristics of the 5 patients are presented in Table 1. The patients were aged between 51 and 79 years, and included 4 females diagnosed with rheumatoid arthritis (RA) and 1 male diagnosed with systemic sclerosis (SSc). Of the 5 patients, 2 had a history of close contact with COVID-19 patient. Moreover, out of the 5 patients, 3 had a concomitant presence of hypertension and pulmonary diseases (old tuberculosis and bronchiectasis $(n=1)$, chronic bronchitis $(n=1)$, and chronic obstructive pulmonary disease $(n=1))$. Amongst these patients, the most frequently observed symptom was fever (4/5), and cough (4/5) followed by dyspnea (2/5), and fatigue $(1 / 5)$. None of the patients had diarrhea. On laboratory investigations, 2 patients had leukopenia and lymphopenia. The levels of CRP were raised in 3 patients ( $>3 \mathrm{mg} / \mathrm{L}$ ), while the PCT was normal in all the patients $(0-0.05 \mathrm{ng} / \mathrm{mL})$. Due to the increased disease severity, the levels of IL-6 (0.1-2.9 pg/ $\mathrm{mL})$ and IL-10 $(0.1-5 \mathrm{pg} / \mathrm{mL})$ were found to be raised in 3 and 2 patients, respectively. Chest computerized tomography (CT) revealed a patchy ground glass opacity in the lungs of all the patients. In the later stage, it revealed a strip shadow. In the patient with SSc, chest $\mathrm{CT}$ revealed a grid shadow, which may be related to the interstitial pneumonia, as shown in Fig. 1.

The male patient with SSc had been receiving Methylprednisolone ( $8 \mathrm{mg} /$ day) for the last 1 year, which was continued during the entire period of hospitalization, while a female patient with RA was receiving Infliximab (a tumor necrosis factor (TNF- $\alpha$ ) blocker, $200 \mathrm{mg}$ once every 2 months) and Methotrexate (10 mg/week), but on hospitalization, Infliximab was replaced with Leflunomide $(10 \mathrm{mg} /$ day) and Methotrexate (10 mg/week) was continued. However, amongst the other three female RA patients, one had stopped her medication 1 month prior to the hospitalization, while the other two had stopped their medication for several years.

Following the hospitalization, based on the underlying condition, all the patients were given different treatments. However, all these patients were prescribed antiviral treatment, including Arbidol, Oseltamivir, Lopinavir/Ritonavir, Interferon (IFN- $\alpha$ ), and Ribavirin. Amongst them, four patients were given antibacterial therapy, including Levofloxacin, Moxifloxacin, Ceftriaxone, PiperacillinTazobactam, and Meropenem. During the hospital stay, the condition of two female patients remained the same (i.e., mild COVID-19). However, other two female patients progressed to the severe COVID-19, and the male patient worsened to the critical COVID-19. After 3 days of noninvasive ventilator treatment, the critically ill male patient was treated with high-flow oxygen inhalation and was also prescribed Methylprednisolone ( $40 \mathrm{mg} /$ day, 5 days) combined with intravenous Immunoglobulin (IVIg, 10 g/day, 3 days). After 1431 days of hospitalization, all the patients recovered well. In all the patients, the SARS-CoV-2 nucleic acid test was performed twice and was found to be negative, and thus, the 

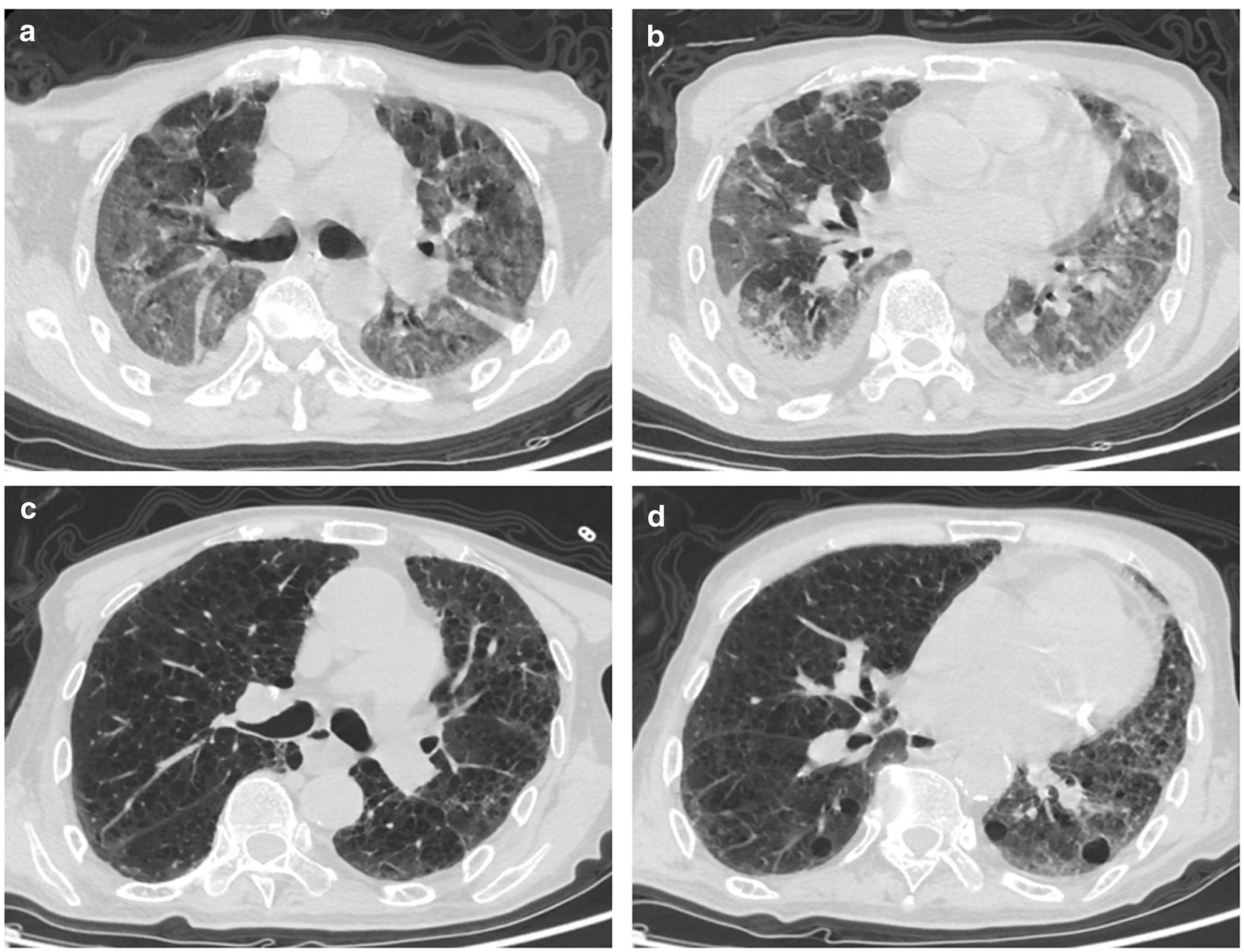

Fig. 1 Chest computerized tomography (CT) scans (transverse plane) of the 5th patient (79-year-old male). a, b 7 days after the symptom onset: The texture of both lungs is disordered, the transparency is increased, some of them have reticular changes, multiple patchy ground glass

patients were discharged from the hospital. Moreover, all the patients were isolated at home for 2 weeks without obvious discomfort, except the 2th patient, who complained of chest pain on deep breathing and followed the doctor's instructions for functional exercise now.

\section{Discussion}

The patients with rheumatic immune diseases have immune dysfunction and may be more susceptible to contract the SARS-CoV-2 infection, and swiftly deteriorate into a severe type [5]. In this case report, we present 5 cases of COVID-19 with concomitant presence of rheumatic immune diseases, thus accounting for $0.88 \%$ of the total confirmed COVID-19 patients admitted in our hospital, which may underestimate the incidence of rheumatic diseases in patients with COVID19. It may be related to the omission on the part of the medical density shadow and fuzzy edge are seen in both lungs. c, $\mathbf{d} 18$ days after the symptom onset: Similar findings as a and $\mathbf{b}$, however, with a few patchy and thin shadows, which are significantly smaller than the previous

staff during the process of history taking. Various studies have reported that the overall incidence rate of COVID-19 is higher in males than in females (58.1\% vs $41.9 \%$ ) [6], which may be related to differences in innate immunity and sex hormones. However, in this case report, 4 patients were female, accounting for $80 \%$ of the cases and this may be related to the high incidence of RA in females.

Amongst the five patients, the severity of COVID-19 was mild and severe in two patients each, and critical in one patient. Thus, the proportion of patients with severe and critical COVID19 was $60 \%$, and two of them were complicated by the baseline pulmonary diseases. One of the RA patients was treated with Methotrexate during the entire period of hospitalization, and her condition remained stable. According to a study, coadministration of Methotrexate and Hydroxychloroquine did not result in an increased risk of hospitalization due to infection [7]. In the present report, a 79-year-old male patient with SSc progressed to critical COVID-19, and this may be explained by 
old age, low immune function, corticosteroid therapy, and concomitant presence of baseline diseases [8,9]. Prior to the hospitalization, this patient was not diagnosed as a case of interstitial pneumonia. However, during hospitalization, his repeated chest CT examination revealed the change of grid shadow, thus suggestive of interstitial pneumonia. This may be related to the alveolar injury that occur in patients with severe or critical COVID-19, or SSc with prior interstitial pneumonia [10]. While, the other two RA patients, who had discontinued their medication, progressed to severe COVID-19. This suggests that drug withdrawal may be a risk factor for disease progression, if the disease has not been stable over a long period.

Various studies have reported that the patients with severe COVID-19 have immune dysfunction, decreased lymphocyte count, and increased levels of cytokines [11], which are consistent with our findings. Amongst the 5 patients, the levels of IL-6 were raised in severe $(n=2)$ and critically ill $(n=1)$ patients. Similarly, the levels of IL-10 were raised in severe $(n=$ $1)$ and critically ill $(n=1)$ patients. In these patients, we prescribed Thymosin (IM, $10 \mathrm{mg} /$ day) to enhance immunity and Hydroxychloroquine (PO, $400 \mathrm{mg} /$ day) to regulate the immunity [12]. These treatments were used for the management of inflammatory storm and immune regulation in patients with severe and critical COVID-19. Moreover, biological agents such as IL-6 blocker (IVIg, $400 \mathrm{mg}$ ) and plasma exchange (400 $\mathrm{ml}$, one or two times) were used in the treatment of severe and critically ill patients.

In summary, we described the characteristics of five rheumatic immune disease patients with the concomitant presence of COVID-19. The patients with rheumatic immune diseases are more likely to progress into severe or critical COVID-19. These two diseases overlap and influence each other in terms of pathogenesis and pharmacological management. Further research is required to understand the entire spectrum of clinical features, diagnostic techniques, and treatment approaches in the management of rheumatic immune disease patients with the concomitant presence of COVID-19.

Availability of data and material The datasets generated during and/or analyzed during the current study are available from the corresponding author on reasonable request.

\section{Compliance with ethical standards}

\section{Disclosures None.}

Ethics approval The experimental protocol was established, according to the ethical guidelines of the Helsinki Declaration, and was approved by Ethics Committee of The Central Hospital of Xiaogan (No. XGLY202003-28), China.

Consent to participate Written informed consent was obtained from patients.
Consent for publication Written informed consent was obtained from patients for publication.

\section{References}

1. Zhou P, Yang XL, Wang XG, Hu B, Zhang L, Zhang W, Si HR, Zhu Y, Li B, Huang CL, Chen HD, Chen J, Luo Y, Guo H, Jiang RD, Liu MQ, Chen Y, Shen XR, Wang X, Zheng XS, Zhao K, Chen QJ, Deng F, Liu LL, Yan B, Zhan FX, Wang YY, Xiao GF, Shi ZL (2020) A pneumonia outbreak associated with a new coronavirus of probable bat origin. Nature 579:270-273. https://doi.org/10.1038/ s41586-020-2012-7

2. Wang D, Hu B, Hu C, Zhu F, Liu X, Zhang J, Wang B, Xiang H, Cheng Z, Xiong Y, Zhao Y, Li Y, Wang X, Peng Z (2020) Clinical characteristics of 138 hospitalized patients with 2019 novel coronavirus-infected pneumonia in Wuhan, China. JAMA 323: 1061. https://doi.org/10.1001/jama.2020.1585

3. Mehta P, McAuley DF, Brown M, Sanchez E, Tattersall RS, Manson JJ (2020) COVID-19: consider cytokine storm syndromes and immunosuppression. Lancet 395:1033-1034. https://doi.org/ 10.1016/s0140-6736(20)30628-0

4. National Health Commission (2020) Diagnosis and Treatment Protocol for Novel Coronavirus Pneumonia (Trial Version 7). http://en.nhc.gov.cn/2020-03/29/c_78469.htm. Accessed 29 Mar 2020

5. Favalli EG, Biggioggero M, Meroni PL (2014) Methotrexate for the treatment of rheumatoid arthritis in the biologic era: still an "anchor" drug? Autoimmun Rev 13:1102-1108. https://doi.org/10. 1016/j.autrev.2014.08.026

6. Wang C, Horby PW, Hayden FG, Gao GF (2020) A novel coronavirus outbreak of global health concern. Lancet 395:470-473. https://doi.org/10.1016/s0140-6736(20)30185-9

7. Singh S, Facciorusso A, Dulai PS, Jairath V, Sandborn WJ (2020) Comparative risk of serious infections with biologic and/or immunosuppressive therapy in patients with inflammatory bowel diseases: a systematic review and meta-analysis. Clin Gastroenterol Hepatol 18:69-81.e63. https://doi.org/10.1016/j.cgh.2019.02.044

8. Listing J, Gerhold K, Zink A (2013) The risk of infections associated with rheumatoid arthritis, with its comorbidity and treatment. Rheumatology (Oxford) 52:53-61. https://doi.org/10.1093/ rheumatology $/ \operatorname{kes} 305$

9. Russell CD, Millar JE, Baillie JK (2020) Clinical evidence does not support corticosteroid treatment for 2019-nCoV lung injury. Lancet 395:473-475. https://doi.org/10.1016/s0140-6736(20)30317-2

10. Yang X, Yu Y, Xu J, Shu H, Xia J, Liu H, Wu Y, Zhang L, Yu Z, Fang M, Yu T, Wang Y, Pan S, Zou X, Yuan S, Shang Y (2020) Clinical course and outcomes of critically ill patients with SARSCoV-2 pneumonia in Wuhan, China: a single-centered, retrospective, observational study. Lancet Respir Med 8:475-481. https:// doi.org/10.1016/s2213-2600(20)30079-5

11. Favalli EG, Ingegnoli F, De Lucia O, Cincinelli G, Cimaz R, Caporali R (2020) COVID-19 infection and rheumatoid arthritis: faraway, so close! Autoimmun Rev 19:102523. https://doi.org/10. 1016/j.autrev.2020.102523

12. Nicastri E, Petrosillo N, Bartoli TA, Lepore L, Mondi A, Palmieri F D’Offizi G, Marchioni L, Murachelli S, Ippolito G, Antinori A (2020) National Institute for the infectious diseases "L. Spallanzani”, IRCCS. Recommendations for COVID-19 clinical management. Infect Dis Rep 12:8543. https://doi.org/10.4081/idr. 2020.8543

Publisher's note Springer Nature remains neutral with regard to jurisdictional claims in published maps and institutional affiliations. 